\title{
Substrate Effects on the Phonon Response of Individual Dielectric Nanostructures
}

Ka Yin Lee ${ }^{1}$, Elliot Beutler ${ }^{2}$, David Masiello ${ }^{2}$ and Maureen Joel Lagos ${ }^{3}$

${ }^{1}$ Department of Materials Science and Engineering, McMaster University, Hamilton, Ontario, Canada, Canada, ${ }^{2}$ Department of Chemistry, University of Washington, Seattle, USA, United States,

${ }^{3}$ Department of Materials Science and Engineering, McMaster University, Hamilton, Canada, Canada

With the advent of new high-energy resolution monochromators, spatially-resolved electron probes are gaining importance as an infrared spectroscopy tool to characterize materials at the nanoscale. For instance, vibrational EELS signal has been used to measure local properties in individual nanostructures such as temperature [1,2], spatial distribution of phonons [3], vibrational signatures in biomaterials [4], etc. However, the interpretation of this EELS data can present challenges, requiring a deeper understanding of several physical processes such as electron screening, $f$-sum rules, thermal population effects [5], and resonant substrate effects [6]. In this work, we present a detailed experimental and theoretical study of the phonon coupling between a single dielectric sphere and a thin support. This study highlights the relevant role of the substrate on the surface phonon response of composite object-substrate systems, thus aiding in performing accurate measurements and correct data interpretation.

We performed spatially-resolved EELS studies using a Nion UltraStem equipped with a monochromator using $\mathrm{a} \sim 1.5-2 \AA$ probe with energy spread of $\sim 9 \mathrm{meV}$, at $60 \mathrm{kV}$. The combination of high spatial and energy resolution has allowed the detection of surface/bulk phonon excitations over a wide variety of individual nanostructures [1-8].

Specifically, we probed $\mathrm{SiO}_{2}$ spheres of different radius $(150-500 \mathrm{~nm})$ that are partially suspended from a thin substrate - a configuration that minimizes sphere-substrate interaction (inset Fig. 1a). The EEL spectra revealed two main resonances located within the two Reststrahlen bands of the material (Fig. 1a) that increase in intensity as the sphere size increases. Each EELS peak is due to the excitation of the main dipole mode and other higher energy multipole modes, resulting in slightly asymmetric peaks (Fig. 1b). The dipole mode dominates the scattering signal ( $>45 \%$ for spheres below $250 \mathrm{~nm}$ in size). Minor redshifts $(<2 \mathrm{meV})$ were observed for increasing sphere sizes, revealing the limited tunability of the phonon polaritonic response as a function of sphere size. The phonon polariton behavior of thin films was also investigated, indicating that their responses are due to Fuchs-Kliewer modes, in agreement with previous experimental results [7].

Each composite sphere-substrate system was probed to study the sphere-substrate phonon interaction. A combination of several materials $\left(\mathrm{SiO}_{2}, \mathrm{Si}_{3} \mathrm{~N}_{4}\right.$, and $\left.a-\mathrm{C}\right)$ was selected to conduct a systematic study of the sphere-substrate behavior. Figure 2a shows EEL spectra of several composite systems, including spectroscopic results obtained from isolated thin films. Our theoretical modeling elucidates how the total loss probability of the coupled system is expressed by a combination of contributions from the sphere's and substrate's surface phonon modes [6]. Subtraction of substrate contributions from the EEL spectra clarifies interpretation of the coupled modes and resonance shifts as being due to hybridization $[7,8]$ and image charge effects, as revealed by the scattering intensities at $\sim 125 \mathrm{meV}$ in Figure $2 \mathrm{~b}$. The formation of these new coupled modes evidences the substrate-sphere interaction. 
Furthermore, we studied the effects of the substrate thickness $(5-15 \mathrm{~nm})$ on the scattering intensity (Fig. 2c). We note that the phonon scattering intensity can be significantly reduced for thicker films, as a result of the strong interaction of the phonon modes with their image charge. Spectral shifts of the main resonances as a function of substrate thickness were also observed (Fig. 2c). A comparison of scattering intensity from spheres on amorphous carbon with partially suspended spheres revealed that a decrease of scattering intensity of up to $50 \%$ is possible for spheres of radius larger than $250 \mathrm{~nm}$.

In summary, we have investigated the surface phonon interactions between a single dielectric sphere and a resonant thin dielectric film support. We found that the surface phonon behavior can be drastically affected by the presence of the substrate with new coupled modes generated due to phonon mode mixing. Our results extend the understanding of surface phonon coupling between nanostructures and their support, revealing the importance of the role played by a substrate in quantitative phonon EELS analysis, which is essential to the accurate measurement of physical properties such as infrared energy-transferred processes.

Acknowledgement: For financial support, M.J.L. acknowledges the NSERC under a Discovery Grant. Work at the University of Washington was supported by the U.S. Department of Energy (DOE), Office of Science, Office of Basic Energy Sciences (BES), Materials Sciences and Engineering Division under Award DE-SC0018040 (D.J.M.). We also acknowledge Philip Batson and Gianluigi Botton for discussions regarding coupling processes. Corresponding author: mjlagos@ mcmaster.ca
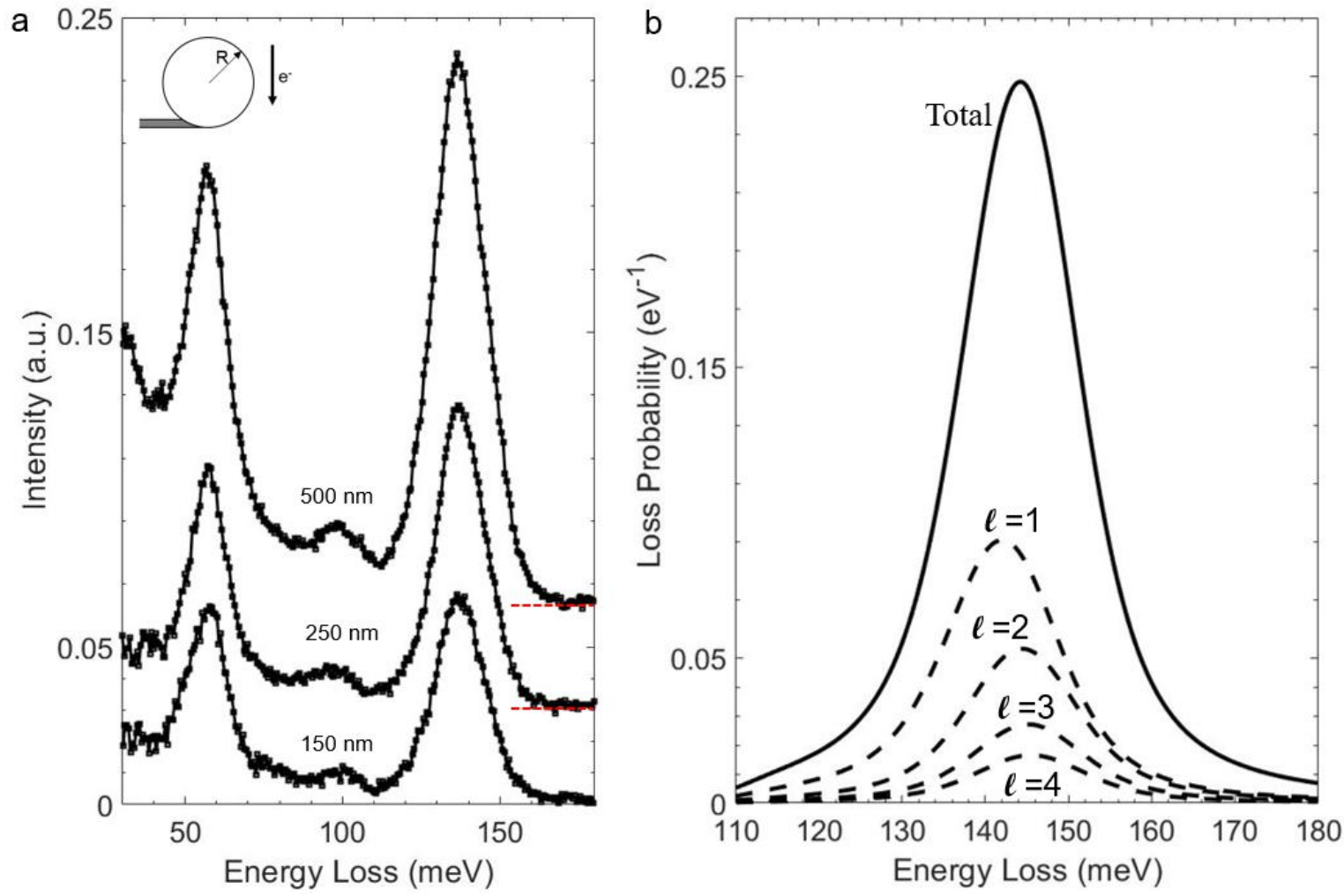

Figure 1. (a) Background subtracted experimental EELS spectra obtained for partially suspended $\mathrm{SiO} 2$ spheres. Inset shows a schematic of the aloof excitation of a sphere (radius $=\mathrm{R}$ ). (b) Simulated EELS loss probabilities for an isolated $\mathrm{SiO} 2$ sphere $(\mathrm{R}=500 \mathrm{~nm}), \boldsymbol{\ell}=1$ corresponds to the dipolar mode, other $\ell$ 's correspond to higher energy multipole modes. 
a

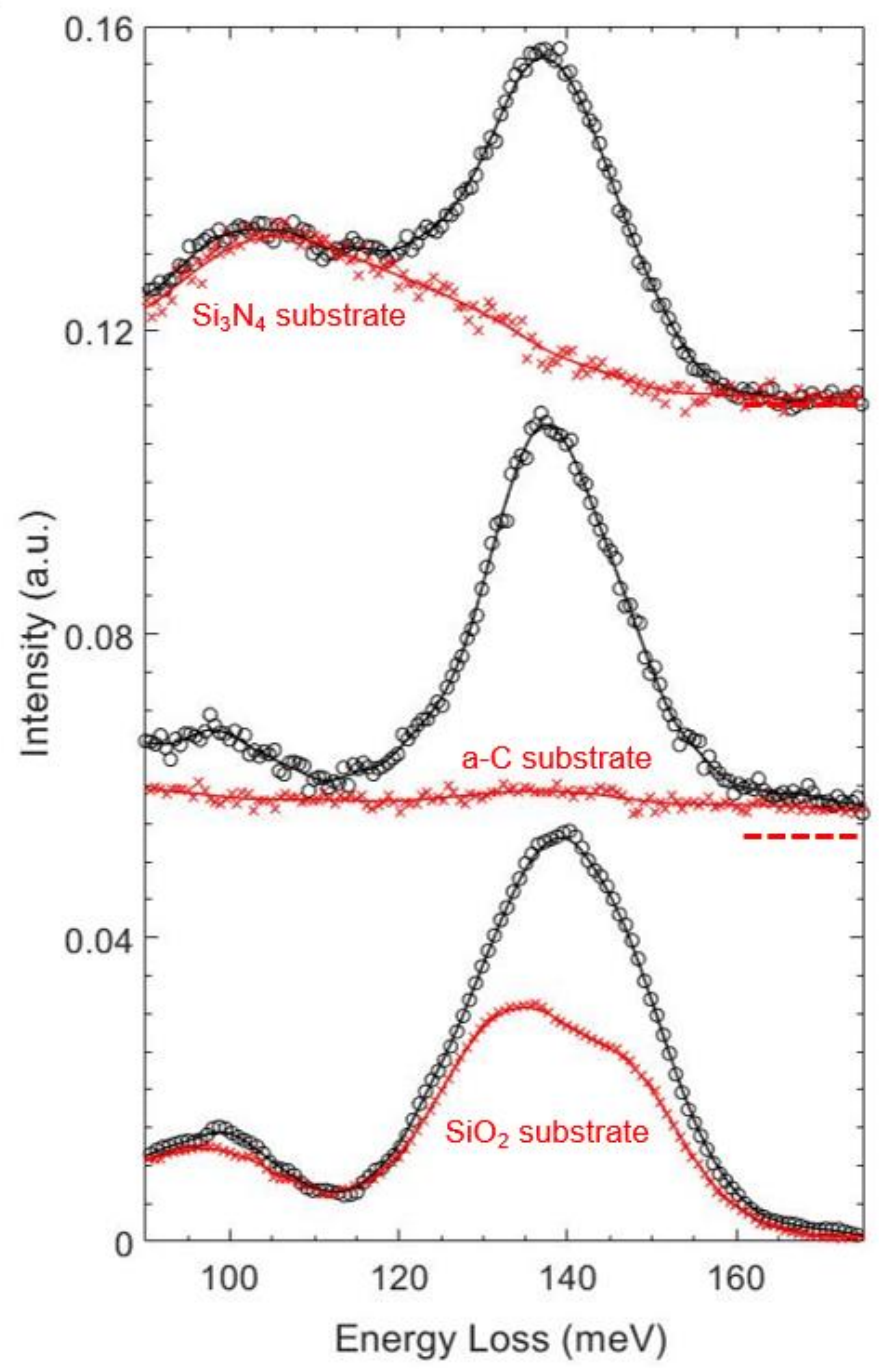

b

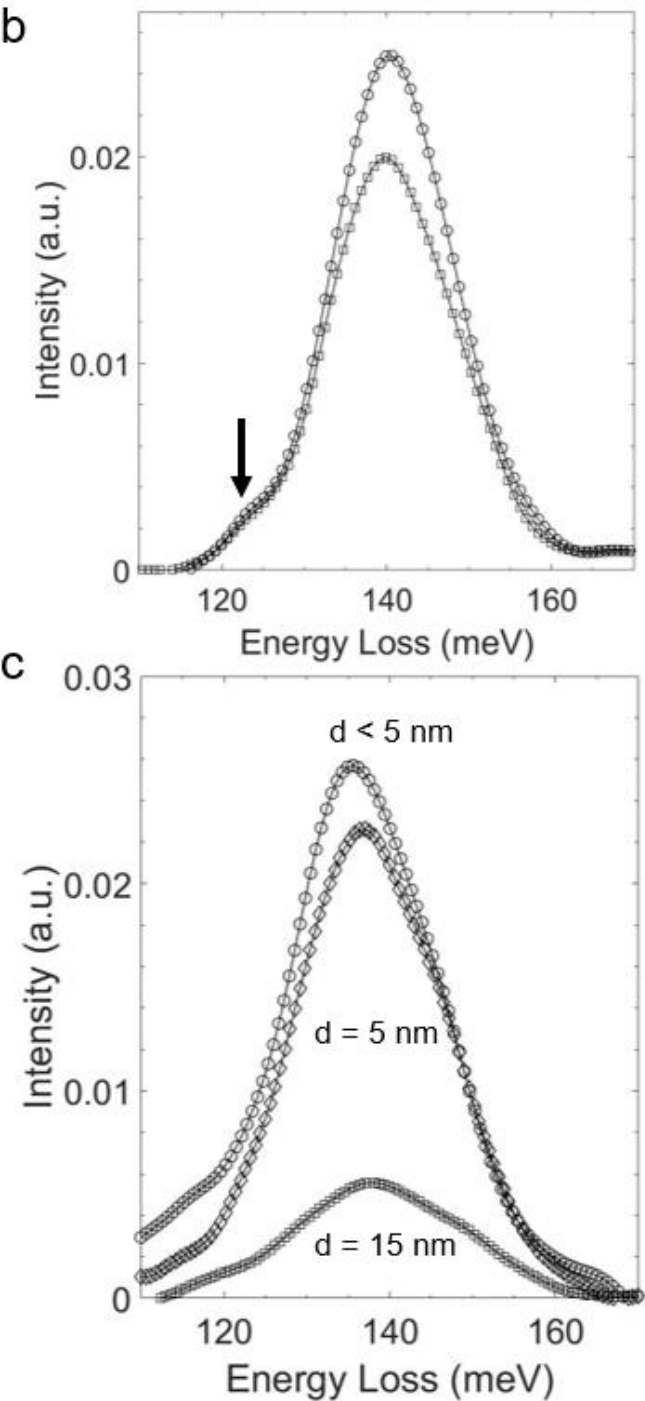

Figure 2. (a) EEL spectra acquired from different sphere-substrate systems. Red curves show EEL spectra for different isolated thin substrates. (b) Subtracted EEL spectra for $\mathrm{SiO} 2$ spheres on $\mathrm{SiO} 2$ substrates at different impact parameters (5 and $10 \mathrm{~nm}$ ). Arrow indicates coupled modes. (c) Subtracted EEL spectra for $\mathrm{SiO} 2$ spheres on amorphous carbon substrate system of different substrate thickness $\mathrm{d}$.

\section{References}

[1] M. J. Lagos, P. E. Batson, NanoLetters 18 (2018) 4556.

[2] J. C. Idrobo, et al, Phys. Rev. Lett. 120, 095901 (2018).

[3] M. J. Lagos, et al, Nature 543 (2017) 529.

[4] P. Rez, et al, Nature Communications 7, 10945 (2016)

[5] P. E. Batson, et al, Microscopy and Microanalysis 24, 412 (2018).

[6] E. K. Beutler, et al, submitted to Phys. Rev. B (2021).

[7] M. J. Lagos, et al, Microscopy 67, (2018) i3.

[8] H. Lourenço-Martins, et al, Phys. Rev. X 7, 041059 (2017). 